\title{
Violation of Bans on Tobacco Advertising and Promotion at Points of Sale in Viet Nam: Trend from 2009 - 2015
}

\author{
Le Thi Thanh Huong ${ }^{1 *}$, Tran Khanh Long ${ }^{1 *}$, Phung Xuan Son ${ }^{1}$, Do Phuc Huyen ${ }^{1}$, \\ Phan Thuy Linh', Nguyen Ngoc Bich', Nguyen Xuan Lam², Le Vu Anh², Tran \\ Thi Tuyet-Hanh ${ }^{1}$
}

\begin{abstract}
Comprehensive bans on tobacco advertising and promotion were introduced through tobacco control legislation in Viet Nam, but it has been established that violations of the bans are very common. This study was conducted to explore the trend in violations of bans on tobacco advertising and promotion at points of sale in Viet Nam in the past six years and to explore any differences in the violation situations before and after the Law on Tobacco Control came into effect on 1st May 2013. Quantitative data were collected through observation of violations of the bans on tobacco advertising and promotion at points of sale in 10 provinces throughout Viet Nam in four survey rounds $(2009,2010,2011$, and 2015). Variation in violation prevalence over time was examined by chi-square test using a Bonferini method. Binary logistic regression was employed to identify the factors that may have influences on different types of violation. A level of significance of $p<0.05$ was used for all tests in this article. The most common form of violation was the display of more than one pack/one carton of a cigarette brand. Violation of bans on tobacco advertising increased while violations on promotion ban and on displaying tobacco decreased through time. Some factors associated with the tobacco advertising and promotion bans included surveyed years, types of points of sale, regions and areas where the points of sale were located. The enforcement of the bans did not improve even after the issuance and the enactment of the Law on Tobacco Control. This suggests that the monitoring and enforcement of bans on tobacco advertising and promotion at points of sale should be strengthened. Penalties should be strictly applied for violators as indicated in the current tobacco control legislation.
\end{abstract}

Keywords: Violation - tobacco - advertising - promotion - points of sale - Viet Nam

Asian Pac J Cancer Prev, 17 Tobacco Prevention and Control in Vietnam Suppl, 91-96

\section{Introduction}

Advertising is defined in the Vietnamese Law of Advertising as "the employment of various means in order to present the public with the profitable products, goods and service; non-profitable products and services; organizations and individuals trading and providing the presented products, goods and services, except for news, social policies; personal information" (VN National Assembly, 2012a).

Research studies have shown that the advertisement and promotion of tobacco products by the tobacco industry (TI) contributed to the increased number of smokers (Cummings et al., 1991). Tobacco advertising and promotion (TAP) was also blamed for the decreasing efforts of quitting among current smokers who were trying to quit (SEATCA, 2008). These also made people who had quit smoking continue to smoke and were the cause for $34 \%$ of first initiation trials of smoking among youth and children (Cummings et al., 1991).

Due to the comprehensive bans on TAP in many other countries, it is rare to see any violations in mass media such as television, radio, newspapers, etc. However, the tobacco industry has targeted its TAP at points of sale (POSs) using different methods such as billboards, posters, brand colors (ashtrays or parasols with logos) (Kin et al., 2010; HSPH, 2012), non-tobacco products carrying tobacco brand names, such as Davidoff coffee, Camel adventure gear, perfumes and luxury products by Dunhill (Kin et al., 2010), or Vinataba candy in Vietnam etc. (HSPH, 2012). Other promotional activities at POSs are also tactics of the tobacco industry, such as free coupons, free gifts/ samples, price discount, sample distribution of cigarettes, person-to-person promotion (Kin et al., 2010; Sinha et al., 2014; Huong et al., 2015) or promotional girls (HSPH, 2012; Huong et al., 2015). With these activities, the TI is trying to reach their various target groups among communities, such as women (Kin et al., 2010; Brown- 
Johnson et al., 2014), youth, poor people (Kin et al., 2010) and therefore negatively affect tobacco control efforts in different countries.

In Viet Nam, bans on TAP had been issued and came into effect even before the issuance of the Law on Tobacco Control in 2012. In 2005, as a result of the Government's ratification of the World Health Organization Framework Convention on Tobacco Control (WHO FCTC) in 2004, a guideline for implementing the bans on TAP was issued by the Ministry of Culture and Information of Vietnam (VN Ministry of Culture and Information, 2005). In 2012, the Law on Tobacco Control was issued and it was enacted on May 1, 2013. Article 9 of the Law stated that "tobacco advertising and marketing is prohibited in any forms". Besides, the Law only allowed the display of no more than one pack/one carton of a tobacco brand it its 25th article (Viet Nam National Assembly, 2012b). Tobacco advertising is also prohibited by the Law on Advertisement (Viet Nam National Assembly, 2012a). If violate these bans on TAP by the two Laws, financial penalties will be applied to violators with the amount up to 1 or 2 million Vietnam Dong (VND) with the violations of displaying tobacco products or up to 20 or 30 million VND with the violations on marketing tobacco products to the public (The Government of Viet Nam, 2013b). Any violations in tobacco advertising will be given a penalty of 20 to 30 million VND, as stated in the Decree number 158/2013/ ND-CP (The Government of Viet Nam, 2013a).

However, there have existed a reluctance in applying penalties for violators of TAP at POSs in Viet Nam, as indicated by the study conducted by the Hanoi School of Public Health (HSPH, 2013) and therefore, the violations of TAP bans were relatively high, especially at POSs. Before the issuance of the Law on Tobacco Control, the prevalence of violations on TAP at POSs in Vietnam ranged from around $13 \%$ to approximately $25 \%$ of all surveyed POSs, and the most common violation was the display of more than one pack/one carton of a tobacco brand - which accounted for more than $90 \%$ of all POS surveyed (Anh et al., 2011; HSPH, 2012). In the year 2015, the HSPH and the Vietnam Public Health Association (VPHA) jointly conducted the survey on TAP violations in Viet Nam to examine any possible violations of TAP bans after the Law on Tobacco Control came into effect in May 2013.

This article aims to show the trend of TAP ban violations in Viet Nam over four time points (2009, 2010, 2011 and 2015) and to identify factors associated with types of TAP ban violations. Results gained from this study would be helpful in providing scientific evidence for advocacy for better enforcement of TAP bans in Viet Nam.

\section{Materials and Methods}

\section{Data source}

This study uses data from two related projects, including: (1) "Towards zero tobacco advertising, promotion and sponsorship in Viet Nam" which provided the data on TAP violations at 1,390 POSs in 10 provinces throughout Viet Nam from 2009 to 2011 and (2) "Communication and Advocacy for the effective implementation of regulations for zero tobacco advertising, promotion and sponsorship in Viet Nam" with the contribution of data from Viet Nam Public Health Association (VPHA), which contributed the data of 1,416 POSs in 10 provinces in 2015.

The surveys collected data in 10 provinces/cities throughout Viet Nam. For the surveys implemented in 2009, 2010, and 2011, the 10 provinces/cities included Lao Cai, Yen Bai, Hai Duong, Thai Binh (in the North), Ha Tinh, Da Nang, Khanh Hoa (in the Central), and Dong Nai, Ho Chi Minh City, Dong Thap (in the South). For the survey in 2015, the 10 provinces/cities were Ha Noi, Hai Phong, Hai Duong, Thai Binh (in the North), Ha Tinh, Da Nang, Khanh Hoa (in the Central), Bac Lieu, Ho Chi Minh City, and Dong Thap (in the South). At each provinces, 10 most crowded streets in urban area and five most crowded streets in rural area were selected. At each selected street, 8 - 10 POSs were chosen randomly, assuring POSs separated each other for at least five house/shop address numbers. A similar tools and data collector groups from Hanoi School of Public Health and VPHA were used in the surveys. A data collection training workshop was organized for all data collectors to ensure all of them could use the data collection tools properly.

\section{Definition of dependent variables used in this article}

i). Violation of the advertising bans at POSs: The violation of tobacco advertising bans was recorded at POSs if data collectors observed one or more of the following activities to present tobacco products to the general public at POSs, such as using counter with logo/ symbol/ brand name/ color of tobacco products; Poster/ billboard with logo/ symbol/ brand name/ color of tobacco products outside of POSs; Ribbons/ umbrellas with logo/ symbol/color/ brand name of tobacco products; Tobacco display shelf/shelves with logo/ symbol/ brand name/ color of tobacco products; Objects with logo/ symbol/ brand name of tobacco products (lighters, ash-trays etc.);

ii). Violation of the ban on tobacco promotions at POSs: POSs were considered as violating tobacco promotion bans if the following activities were recorded during the data collection: discount price, free gifts (lighter, USB, key hanger, cap...), free coupons;

iii). Violation of displaying tobacco products at POSs: Any POSs recorded with the criteria of displaying more than one pack/one carton of a tobacco brand were considered as violation;

iv). Violation of all types of TAP bans at POSs: POSs were considered as violating all types of TAP bans if the POSs were recorded as violating all three types, including advertising, displaying and promotion bans.

\section{Definition of independent variables used in this article}

(1) Year: Four periods of time in which the data collection were performed, including 2009, 2010, 2011 and 2015

(2) Type of POSs: Three types of POSs, included coffee shops/pubs/karaoke; convenient stores/groceries 
and push - carts

(3) Geographic: region (North, Centre, South) and Area (urban, rural)

\section{Tool for data collection}

Tool for data collection of these two projects was an observational checklist to record any violations of TAP bans at surveyed POSs. The tool was developed during the year 2009, with the technical assistance of the Tobacco Free Kids, then was piloted at POSs in Hanoi and was adjusted before the data collection. The same tool was used for observation of TAP ban violations at surveyed POSs during the next data collection rounds in 2010, 2011 and 2015.

\section{Statistical analysis}

Descriptive and statistical analyses with percentages and $95 \%$ confidence interval (CI) were calculated using the software IBM SPSS version 22.0. Differences of the violation prevalence throughout the four surveys were examined by chi-square test using the Bonferini method. Binary logistic regression was applied to identify any factors (region, type of POSs, area, time of data collection) associated with the different types of violation. The level of significant 0.05 was used for all tests in this article.

\section{Ethical consideration}

Ethical clearance of the 2015 study was approved by the Institutional Review Board of the Hanoi School of Public Health (No 289/2014/YTCC-HD3 on 28 October 2014). The use of data sources from 2009 to 2011 was approved by the Director Board of the Hanoi School of Public Health.

\section{Results}

Data on general information of the surveyed POSs are presented in Table 1. In total, there were 1,390 POSs surveyed in the period from 2009 through 2011 and 1,416 POSs surveyed in the year 2015. The main types of POSs observed in all four surveys were convenient stores/groceries, followed by café/pubs/karaoke bars. The distributions of POSs across regions (North, Central, and South) were not much different, with a little higher proportion in the North compared to those in the Central and the South. Around more than two-thirds of the POSs were located in the urban areas. There were no significant differences between types of POSs, POSs by regions, by areas and by time from 2009 to 2015 .

The trends of TAP bans violation types are represented in Figure 1. As indicated, the overall trend of violation seems to decrease significantly by years for almost all types of violations, except for the reverse trend of the violation of tobacco advertising bans $(\mathrm{p}<0.001)$. The decreasing trends were most significant from 2011 to 2015 , while the increasing trends of the violation in tobacco advertising bans were also observed from 2011 to 2015 . Although the results showed promisingly decreasing trends, it can be seen from Figure 1 that the prevalences of violating the bans on tobacco packages/carton display were still very high, with nearly all surveyed POSs violated the bans during 2009 - 2011 and althoughdecreased in 2015, this prevalence was still at $86.9 \%$. For the violation of tobacco advertising bans, there had been a significant increased prevalence of violation in $2015(40.1 \%)$ as compared to those in the previous years $(2009,2010$ and 2011).

To identify factors associated with different types of the violations on TAP bans, we used some independent factors such as time (years), types of POSs, region (North, Centre and South), and area (urban, rural) to run the

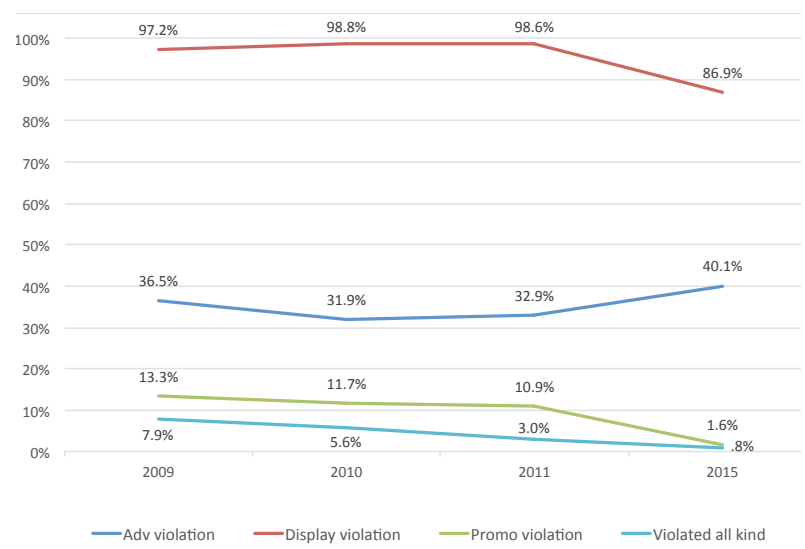

Figure 1. Types of Violations of TAP Bans by Time

Table 1. General Information of the Study Samples

\begin{tabular}{lccccccccc}
\hline \multicolumn{1}{c}{ Type of POSs } & \multicolumn{2}{c}{2009} & \multicolumn{2}{c}{2010} & \multicolumn{2}{c}{2011} & \multicolumn{2}{c}{2015} & $\mathrm{P}$ \\
\hline Convenient stores / groceries & 920 & 66.2 & 969 & 69.7 & 953 & 68.6 & 951 & 67.2 & $\chi^{2}=8.5 ;$ \\
Café/ pubs/ karaoke & 348 & 25 & 289 & 20.8 & 303 & 21.8 & 334 & 23.6 & $\mathrm{df}=6 ;$ \\
Push - carts & 122 & 8.8 & 132 & 9.5 & 133 & 9.6 & 131 & 9.3 & $\mathrm{p}=0.2$ \\
Total & 1390 & 100 & 1390 & 100 & 1389 & 100 & 1416 & 100 & \\
Regions & & & & & & & & & \\
North & 567 & 40.8 & 567 & 40.8 & 567 & 40.8 & 564 & 39.8 & $\chi^{2}=5.6 ;$ \\
Centre & 439 & 31.6 & 439 & 31.6 & 439 & 31.6 & 416 & 29.4 & $\mathrm{df}=6 ;$ \\
South & 384 & 27.6 & 384 & 27.6 & 384 & 27.6 & 436 & 30.8 & $\mathrm{p}=0.5$ \\
Total & 1390 & 100 & 1390 & 100 & 1390 & 100 & 1416 & 100 & \\
Areas & & & & & & & & & \\
Urban & 955 & 68.7 & 989 & 71.2 & 962 & 69.2 & 996 & 70.3 & $\chi^{2}=2.4 ;$ \\
Rural & 435 & 31.3 & 401 & 28.8 & 428 & 30.8 & 420 & 29.7 & $\mathrm{df}=3 ;$ \\
Total & 1390 & 100 & 1390 & 100 & 1390 & 100 & 1416 & 100 & $\mathrm{p}=0.4$ \\
\hline
\end{tabular}


Table 2. Associations between Types of Violations of TAP Bans and Selected Factors*

\begin{tabular}{|c|c|c|c|c|c|c|c|c|}
\hline & \multicolumn{2}{|c|}{ Ads violation } & \multicolumn{2}{|c|}{ Promotion violation } & \multicolumn{2}{|c|}{ Display violation } & \multicolumn{2}{|c|}{ Violation in common } \\
\hline & $\beta$ & OR $(95 \% \mathrm{CI})$ & $\beta$ & OR $(95 \% \mathrm{CI})$ & $\beta$ & OR $(95 \% \mathrm{CI})$ & $\beta$ & $\mathrm{OR}(95 \% \mathrm{CI})$ \\
\hline Intercept & \multicolumn{2}{|r|}{-0.795} & \multicolumn{2}{|r|}{-3.555} & \multicolumn{2}{|r|}{2.229} & \multicolumn{2}{|r|}{-4.582} \\
\hline \multicolumn{9}{|l|}{ Year } \\
\hline 2009 & -0.16 & $\begin{array}{c}0.85 \\
(0.72-1)\end{array}$ & 2.25 & $\begin{array}{c}9.52 \\
(6.13-14.81)\end{array}$ & 1.67 & $\begin{array}{c}5.31 \\
(3.72-7.57)\end{array}$ & 2.32 & $\begin{array}{c}10.16 \\
(5.55-18.61)\end{array}$ \\
\hline 2010 & -0.44 & $\begin{array}{c}0.65 \\
(0.55-0.77)\end{array}$ & 2.07 & $\begin{array}{c}7.89 \\
(5.06-12.31)\end{array}$ & 2.58 & $\begin{array}{c}13.22 \\
(7.88-22.18)\end{array}$ & 1.85 & $\begin{array}{c}6.35 \\
(3.42-11.77)\end{array}$ \\
\hline 2011 & -0.37 & $\begin{array}{c}0.69 \\
(0.58-0.82)\end{array}$ & 2 & $\begin{array}{c}7.36 \\
(4.71-11.5)\end{array}$ & 2.41 & $\begin{array}{c}11.11 \\
(6.88-17.94)\end{array}$ & 1.23 & $\begin{array}{c}3.41 \\
(1.78-6.54)\end{array}$ \\
\hline 2015 & & 1 & & 1 & & 1 & & 1 \\
\hline \multicolumn{9}{|l|}{ Type of POSs } \\
\hline Café/ pubs/ karaoke & -1.56 & $\begin{array}{c}0.21 \\
(0.17-0.26)\end{array}$ & -0.56 & $\begin{array}{c}0.57 \\
(0.43-0.75)\end{array}$ & -0.13 & $\begin{array}{c}0.87 \\
(0.55-1.38)\end{array}$ & -1.15 & $\begin{array}{c}0.32 \\
(0.23-0.44)\end{array}$ \\
\hline $\begin{array}{l}\text { Convenient stores / } \\
\text { groceries }\end{array}$ & -1.64 & $\begin{array}{c}0.19 \\
(0.15-0.25)\end{array}$ & -1.25 & $\begin{array}{c}0.29 \\
(0.2-0.41)\end{array}$ & 0.01 & $\begin{array}{c}1.01 \\
(0.6-1.68)\end{array}$ & -1.84 & $\begin{array}{c}0.16 \\
(0.1-0.26)\end{array}$ \\
\hline Push - carts & & 1 & & 1 & & 1 & & 1 \\
\hline \multicolumn{9}{|l|}{ Region } \\
\hline North & -0.93 & $\begin{array}{c}0.4 \\
(0.34-0.46)\end{array}$ & -0.28 & $\begin{array}{c}0.76 \\
(0.6-0.96)\end{array}$ & 0.07 & $\begin{array}{c}1.08 \\
(0.77-1.51)\end{array}$ & -0.74 & $\begin{array}{c}0.48 \\
(0.33-0.7)\end{array}$ \\
\hline Centre & 0.62 & $\begin{array}{c}1.86 \\
(1.61-2.15)\end{array}$ & -0.03 & $\begin{array}{c}0.97 \\
(0.77-1.23)\end{array}$ & -0.57 & $\begin{array}{c}0.57 \\
(0.41-0.78)\end{array}$ & 0.1 & $\begin{array}{c}1.11 \\
(0.81-1.51)\end{array}$ \\
\hline South & & 1 & & 1 & & 1 & & 1 \\
\hline \multicolumn{9}{|l|}{ Area } \\
\hline Urban & 0.54 & $\begin{array}{c}1.71 \\
(1.49-1.96)\end{array}$ & 0.24 & $\begin{array}{c}1.27 \\
(1.03-1.58)\end{array}$ & -0.11 & $\begin{array}{c}0.9 \\
(0.67-1.2)\end{array}$ & 1.36 & $\begin{array}{c}3.9 \\
(2.51-6.04)\end{array}$ \\
\hline Rural & & 1 & & 1 & & 1 & & 1 \\
\hline
\end{tabular}

binary logistic regression model The results are shown in Table 2. Firstly, it is seen in Table 2 that, the violations on tobacco advertising in 2009, 2010 and 2012 were $15 \%$, $35 \%$ and $31 \%$ lower than that of 2015 , respectively. Pushcarts were the type of POSs with the highest number of violations of tobacco advertising bans, as compared to the other types of POSs. According to area (North, Central, South), the results showed that POSs in the Central had more violations of tobacco advertising bans than did the North and the South. POSs in the urban areas had higher prevalence of violating the tobacco advertising bans than those in the rural areas $(\mathrm{OR}=1.71,95 \% \mathrm{CI}: 1.49-1.96)$. Secondly, for the violation of tobacco promotion bans, it is revealed from Table 2 that the violations of this ban in 2009, 2010 and 2011 were 9.52 (95\%CI: 6.13-14.81), 7.89 (95\%CI: 5.06-12.31) and 7.36 (95\%CI: 4.71-11.5) times higher than that of 2015 , respectively.

Similar to the tobacco advertising violation, for tobacco promotion ban, push-carts were also the POS type with the highest violation. The number of POSs in urban areas that violated the displaying bans was 1.27 times higher than that in the rural area (95\%CI: 1.03-1.58). Thirdly, for the violation of the bans of displaying no more than one pack/one carton of a tobacco brand, it is seen in Table 2 that the violation rates in 2009, 2010 and 2011 were 5.31(95\%CI: 3.72-7.57), 13.22 (95\%CI: 7.88-22.18) and 11.11 (95\%CI: 6.88-17.94) times higher than that in the year 2015. And lastly, for the violation of all types of TAP bans (including tobacco advertising, displaying and promotion bans), it is shown in Table 2 that the prevalence of violation in the years 2009,2010 and 2011 were 10.16 (95\% CI: 5.55-18.61), 6.35 (95\% CI: 3.42-11.77), 3.41 (95\% CI: 1.78-6.54) times higher than that of the year 2015 , respectively. Similar to the violation of tobacco advertising and tobacco promotion bans, push-carts were the POSs with higher violation rates than the other two POS types in the study. It was indicated from Table 2 that POSs in the North had lower violation prevalence of TAP bans than those in the Central and in the South. The prevalence of violated POSs in the urban area was higher than those in the rural area $(\mathrm{OR}=3.995 \% \mathrm{CI}: 2.51-6.04)$.

\section{Discussion}

Although the results of the article showed significantly decreased trends in tobacco promotion and displaying in the four periods of time (Figure 1), it could be concluded that the number of violations of the ban "displaying no more than one pack/one carton of a tobacco brand" was still very high, and the results were consistent with those found in previous surveys (HSPH, 2012; Huong et al., 2015). The decreased trend in tobacco displaying might be partly due to the issuance (June 2012) and enactment (May 2013) of the Law on Tobacco Control and some other sub-law documents on the penalties for the administrative violations in health and advertisement sector.

The high violations of displaying tobacco products at POSs may be partly due to the poor compliance and enforcement of the bans in Viet Nam. In fact, a survey conducted by HSPH in 2012 showed that the POS owners 
had very low awareness of the Law and the TAP bans. POS owners did not regard displaying tobacco products as a mean of tobacco advertising, and the accountable authorities were reluctant in giving penalties to POS owners who violated the TAP bans (HSPH, 2012). The results of this study showed that in contrast with the general decreasing trend of violations of TAP bans, the trend for the violation of tobacco advertising bans increased, especially in the year 2015. As indicated in the study of Huong et al. (2015), the most common types of advertising violation at POSs were posters, dummy tobacco packages or lighting packages. Although these types of violation were also found in the previous surveys in 2009 and 2011 (HSPH, 2012), it was seen that these violations were more popular in 2015 in Viet Nam. Given the fact that the Law on Tobacco Control came into effect in May 2013, the situation of violating the TAP bans in Vietnam did not improve as expected.

As indicated in Table 2, the violations of tobacco advertising and tobacco promotion bans were highest at push-carts as compared to the other types of POSs (café/ pubs/karaoke and convenient stores/groceries), and these results in the four survey rounds were similar with those found in the previous studies of the HSPH (2012) and Huong et al. (2015). Regarding the location area of the surveyed POSs (urban/rural area), the results found in this study were also consistent with the previous studies, with higher violations observed at POSs in urban areas as compared to those in the rural areas (HSPH, 2012; Huong et al., 2015). These results again confirmed the unchanged situation of the violations of TAP bans in Viet Nam before and after the enactment of the Law on Tobacco Control.

The poor compliance of TAP bans in Viet Nam described in this article was similar to what was found in Indonesia, where the TAP ban compliance score only ranged from 0 to 1 (the highest score of compliance was 10) (WHO, 2015). India is another country in Asia that has high prevalence of TAP ban violations, where the most common violation was product showcasing (Goel et al., 2014), which was very similar to the situation in Viet Nam. In contrast, in some countries such as Australia or Canada where tobacco control enforcement was conducted properly, the violations decreased and were lower when being compared with those in other countries such as the United States and the United Kingdom where no such restrictions were applied (Li et al., 2013).

Article 13 of the WHO FCTC requires Parties to the treaty to implement and enforce a comprehensive ban on tobacco advertising, promotion and sponsorship within five years of FCTC ratification (WHO, 2003). However, the ban on TAP in Viet Nam is not considered as "comprehensive" because it still allows for the philanthropic sponsorship of the tobacco industry without announcement on mass media and the display of no more than one pack/ one carton of a tobacco brand (VN National Assembly, 2012b). This "non-comprehensive" legislation creates loopholes for the TI in Viet Nam to exploit these gaps to advertise, to market and to promote their tobacco products to consumers. The possible targets may include current smokers, never-smoke community, women and youth, and this was evident in the United States, where the TI tried to target low socioeconomic status women (Brown-Johnson et al., 2014). "Parties recognize that a comprehensive ban on advertising, promotion and sponsorship would reduce the consumption of tobacco products" (WHO, 2003). This statement was concluded by the WHO FCTC, and was proved with the case of Australia, a country that adopted comprehensive bans on TAP. According to a study conducted in Australia in 2013, the ban in displaying tobacco products at POSs led to a reduction in spontaneous tobacco purchases among smokers (Carter et al., 2015)..

In contrast, partial advertising bans are less effective and provide opportunities for tobacco companies to exploit loopholes of the legislation or to find new solutions and tactics to market their products to various target populations, especially women and children (Tobacco Free Kids), and these situations were witnessed in Viet Nam (HSPH, 2012; Huong et al., 2015), in Indonesia (WHO, 2015) as well as in other ASEAN countries (Kin et al., 2010) and in India (Goel et al., 2014).

We need to acknowledge some limitations of our data. This paper used data from 2 separated surveys in 2009 - 2011 and in 2015. The difference in data collection locations may affect the results. The survey applied only to POSs on the streets, therefore none data from supermarkets, malls or stations was included.

In conclusion, the study showed that there had been a decreased trend in the prevalence of violating the TAP bans in Viet Nam, except for the case of the ban on tobacco advertising. However, the violation rates of TAP bans in the country were still high, especially the violation of displaying no more than one pack/one carton of a tobacco brand though the Law on Tobacco Control in Viet Nam has been issued since June 2012 and enacted since May 1 , 2013. Among the many reasons for the poor compliance and enforcement of the bans on TAP, the low awareness of POS owners on TAP provisions and reluctance in giving penalties for TAP ban violators were probably primary.

It is recommended that more comprehensive bans on TAP should be released in Viet Nam, as suggested by the WHO FCTC. In the mean time, a better compliance and enforcement of TAP bans should be strengthened in the country. It is also suggested that increasing the awareness of the POSs owners on TAP ban is critical. In addition, accountable authorities should be made aware of TAP provisions and should strictly apply penalties to all TAP violators, as specified by the appropriate Law.

\section{Acknowledgements}

We thank the Tobacco Free Kids Action Fund (TFK) for their financial support to conduct the four surveys in 2009, 2010, 2011 and 2015. We also thank the Director Board of the Hanoi School of Public Health for their support and approval for the use of the school's data sources from 2009 to 2011 and 2015; and the Vietnam Public Health Association for their survey data being analyzed in this study. In addition, sincerely thanks were sent to the ten Provincial Public Health Associations of VPHA for their valuable efforts and collaborations during the data collection of the surveys from 2009 to 2015. 


\section{References}

Anh LV, Huong LTT, Hanh TTT, et al (2011). Compliance of tobacco advertising, promotion and sponsorship bans in Vietnam, 2010. Vietnam J Public Health, 20, 43-9.

Brown-Johnson C, LJ England, SA Glantz, et al (2014). Tobacco industry marketing to low socioeconomic status women in the U.S.A. Tob Control, 23, 139-46.

Carter O, Phan T, Mills BW (2015). Impact of a point-of-sale tobacco display ban on smokers' spontaneous purchases: comparisons from postpurchase interviews before and after the ban in Western Australia. Tob Control, 24, 81-6.

Cummings KM, Sciandra R, Lawrence J (1991). Tobacco Advertising in Retail Stores. Public Health Rep, 106, 570-5.

Gilpin EA, VM White, P Pierce (2005). How effective are tobacco industry bar and club marketing efforts in reaching young adults? Tobacco Control, 14, 186-92.

Goel R, R Kumar, P Lal, et al (2014). How compliant are tobacco vendors to India's tobacco control legislation on ban of advertisments at point of sale? A three jurisdictions review. Asian Pac J Cancer Prev, 15, 10637-42.

HSPH (2012). Tobacco advertising, promotion and sponsorship in Vietnam - Results from a three year study 2009-2011 at 10 provinces/ cities in Vietnam. 1-120.

HSPH (2013). Stakeholder' awareness on the Law on Tobacco Control and challenges in the implementation of the ban on tobacco advertising, promotion and sponsorship. 1-45.

Huong LTT, Long TK, Bich NN, et al (2015). The status of the violation of tobacco advertising, promotion at points of sale in selected provinces in Vietnam, 2014-2015. 1-53.

Kin F, Lian TY, Yun YC (2010). How the tobacco industry circumvented ban on tobacco advertising, promotion and sponsorships: observation from selected asean countries. Asian J WTO International Health Law Policy, 5, 449-65.

Li L, Borland R, Fong GT, et al (2013). Impact of point-of-sale tobacco display bans: findings from the international tobacco control four country survey. Health Educ Res, 28, 898-910.

Paynter J, Edwards R (2009). The impact of tobacco promotion at the point of sale: a systematic review. Nicotine and Tobacco Res, 11, 25-35.

Robertson L, C Cameron, R McGee, et al (2016). Point-of-sale tobacco promotion and youth smoking: a meta-analysis. Tob Control, pii, tobaccocontrol-2015-052586.

Sardana M, Goel S, Gupta M, et al (2015). Is Exposure to tobacco advertising, promotion and sponsorship associated with initiation of tobacco use among current tobacco users in youth in India? Asian Pac J Cancer Prev, 16, 6299-302.

SEATCA. 2008. The story of point of sale in the Philipines, Thailand and Vietnam [Online]. Bangkok: Southeast Asia tobacco control alliance.

Sinha D, KM Palipudi, K Oswal, et al (2014). Influence of tobacco industry advertisements and promotions on tobacco use in India: findings from the Global Adult Tobacco Survey 2009-2010. Indian J Cancer, 51, 13-8.

The Government of Viet Nam (2013a). Decree 158/2013/NDCP dated 12 November 2013 regulates the administrative penalties for violations in the area of culture, sport, tourisms and advertisement. Decree number 158/2013/ND-CP.

The Government of Viet Nam (2013b). Decree number 176/2013/ND-CP dated 14 November 2013 regulates the administrative penalties for violations in the health sector. Decree number 176/2013/ND-CP.

Tobacco Free Kids. International issues: Advertising and Promotion [Online]. Washington D.C.: TFK.

VN Ministry of Culture - Sport and Tourism (2008). Circular letter 78/2008/TT-BVHTTDL dated 29 August 2008 on adjusting Circular letter 19/2005/TT-BVHTT dated 12 May
2005 of the Ministry of Culture and Information which guides the implementation of the advertisement decree and the Government's resolution 12/2000/NQ-CP on banning tobacco advertising. Circular letter 78/2008/TT-BVHTTDL.

VN Ministry of Culture and Information (2005). Circular letter 19/2005/TT-BVHTT dated 12 May 2005 about the guideline for implementing the ordinance of advertisement and the Government's resolution number of $12 / 2000 / \mathrm{NQ}-\mathrm{CP}$ on banning tobacco advertising. Circular letter 19/2005/TTBVHTT.

VN National Assembly (2012a). Law on Advertisement. 16/2012/QH13.

VN National Assembly (2012b). Law on Tobacco Control. 09/2012/QH13.

Wakefield M, Germain D, Durkin S, et al (2006). An experimental study of effects on schoolchildren of exposure to point-of-sale cigarette advertising and pack displays. Health Education Res, 21, 338-47.

WHO. (2003). WHO FCTC [Online]. Geneva: WHO.

WHO. (2015). WHO report on the global tobacco epidemic, 2015 - Country profile: Indonesia [Online]. Geneva: WHO. 